\title{
Timing performance of the CMS electromagnetic calorimeter and prospects for the future
}

\author{
Adolf Bornheim ${ }^{* \dagger}$ \\ Lauritsen Laboratory, California Institute of Technology, 1200 E California Blvd., Pasadena, \\ 91125 California, USA \\ E-mail: bornheimehep.caltech.edu
}

\begin{abstract}
The CMS electromagnetic calorimeter (ECAL) is made of 75,848 scintillating lead tungstate crystals arranged in a barrel and two endcaps. The scintillation light is read out by avalanche photodiodes in the barrel and vacuum phototriodes in the endcaps, at which point the scintillation pulse is amplified and sampled at $40 \mathrm{MHz}$ by the on-detector electronics. The fast signal from the crystal scintillation enables energy as well as timing measurements from the data collected in proton-proton collisions with high energy electrons and photons. The single-channel time resolution of ECAL measured at beam tests for high energy showers is better than $100 \mathrm{ps}$. The timing resolution achieved with the data collected in proton-proton collisions at the LHC is discussed. We present how precision timing is used in current physics measurements and discuss studies of subtle calorimetric effects, such as the timing response of different crystals belonging to the same electromagnetic shower. In addition, we present prospects for the high luminosity phase of the LHC (HL-LHC), where we expect an average of 140 concurrent interactions per bunch crossing (pile-up). We discuss studies on how precision time information could be exploited for pileup mitigation and for the assignment of the collision vertex for photons. In this respect, a detailed understanding of the timing performance and of the limiting factors in time resolution are areas of ongoing studies.
\end{abstract}

Technology and Instrumentation in Particle Physics 2014,

2-6 June, 2014

Amsterdam, the Netherlands

\footnotetext{
* Speaker.

${ }^{\dagger}$ on behalf of the CMS Collaboration
} 


\section{Introduction}

The primary goal of the Compact Muon Solenoid (CMS) experiment [1] is to explore particle physics at the $\mathrm{TeV}$ energy scale, exploiting the proton-proton collisions delivered by the Large Hadron Collider (LHC) at CERN [2].

The electromagnetic calorimeter (ECAL), which measures the energy of electrons and photons produced in LHC collisions, is located inside a solenoidal magnet which operates at a field strength of 3.8 T. The ECAL is a hermetic homogeneous calorimeter made of 75848 lead tungstate $\left(\mathrm{PbWO}_{4}\right)$ scintillating crystals: 61200 in the barrel (EB) and 7324 in each endcap (EE). The scintillation decay time of the crystals is comparable to the LHC bunch crossing interval of $25 \mathrm{~ns}$, and typically about $80 \%$ of the light is emitted in $25 \mathrm{~ns}$. For the light detection, the crystals are equipped with avalanche photodiodes (APD) in the barrel and vacuum phototriodes (VPT) in the endcaps. The stability of the time measurement required to maintain the energy resolution is on the order of $1 \mathrm{~ns}$. In addition to the energy measurement, the combination of the scintillation timescale of $\mathrm{PbWO}_{4}$, the electronic pulse shaping, and the sampling rate allows excellent time resolution to be obtained with the ECAL.

Maintaining excellent time resolution is important in CMS in many respects. A precise time measurement and synchronization allows the rejection of backgrounds with a broad time distribution. Such backgrounds include out-of-time proton-proton interactions, cosmic rays, beam halo muons, and electronic noise. Precise time measurement is also exploited in searches for particles predicted by beyond the Standard Model physics, identifying photons from the decay of long-lived new particles, which reach the calorimeter out- of-time with respect to particles traveling at the speed of light from the interaction point.

The performance of the calorimeter has been measured prior to the collisions using test beam electrons, cosmics and beam splash events [3]. The systematic limit of the time resolution for large energy deposits was estimated to be approximately 20 ps from these measurements.

In this document we describe the ECAL timing performance in detail and present further studies on the performance achieved with proton-proton collision data at the LHC accelerator.

During collisions, there are many additional effects which could worsen the performance, like run-by-run variations, inter-calibration effects, energy-dependent systematics, and crystal damage due to radiation. A first measurement of the timing performance and intercalibration of the CMS ECAL during collisions has been reported in [4], where the time resolution was about $190 \mathrm{ps}$ in the barrel and $280 \mathrm{ps}$ in the endcap.

Detailed studies on time resolution are also important for the upgrade of the detector foreseen in 2024, when the LHC is expected to reach peak luminosities of $10^{35} \mathrm{~cm}^{-2} \mathrm{~s}^{-1}$ and it will be necessary to replace some components of the current detectors, especially in the forward region. The increased luminosity will introduce a very high multiplicity of simultaneous events (pile-up), possibly in the range of 140 to 200 interactions per beam crossing. The pile-up of energy deposits coming from different interactions deteriorates the calorimeter performances in terms of energy measurement and particle identification, as individual particles are less isolated. A possible strategy to reduce pile-up effects consists in complementing the calorimeters with an extremely high time resolution capability, which would enable the energy deposits coming from different interaction vertices to be resolved in time. 


\section{The CMS ECAL Detector}

The CMS ECAL detector and its performance during the LHC run I in 2011 to 2012 has been presented in detail at this conference [6]. The detector is optimized for a very good energy resolution of photons and electrons. In the test beam an energy resolution of better than $0.5 \%$ was achieved for high energy electrons of $100 \mathrm{GeV}$ and above. In situ with data from proton-proton collision recorded in 2011 and 2012 an energy resolution of around 1\% has been achieved for photons in the most favorable detector regions [4], representative of the bare calorimeter performance.

Some key elements to achieve this performance are the very precise light collection from the ECAL crystals, the very precise pulse shaping and reconstruction and the very good stability of the detector. The linearity of the energy measurement of the ECAL was found to be better than $1 \%$ in the test beam. The pulses are shaped with a $40 \mathrm{~ns}$ time constant and then digitized at a rate of $40 \mathrm{MHz}$. The analog pulse shapes are uniform and reproducible with a precision of $1 \%$ among all the channels of the ECAL and over long times under the harsh LHC operational conditions. The digitization has a dynamic range of 12 bit and the noise is approximately 1 ADC count, equivalent to about $40 \mathrm{MeV}$. The pulse amplitude reconstruction is based on 10 consecutive samples of which three are used to estimate the pedestal just before the pulse and of the remaining seven samples give are used to estimate the pulse amplitude from a weighted sum of the individual samples. The digitization is performed in electronics mounted on the back of the crystals inside the CMS detector. To synchronize the readout electronics a clock signal is distributed to the respective electronic boards. Sets of $5 \times 5$ crystals are served by a common clock distribution line. We refer to this as a readout unit.

The design goal for the timing precision of the ECAL was in the range of $1 \mathrm{~ns}$. This was considered sufficient to ensure synchronization of the readout with the trigger system and to allow a precise assignment of energies measured in the detector to the bunch crossings of the LHC. Using the ratios of consecutive samples one can however reconstruct the time of the energy deposit in the ECAL crystals with much better precision [3]. In Figure 1 we illustrate the pulse energy and time reconstruction in the ECAL. The time of the energy deposited in a crystal is extracted from the
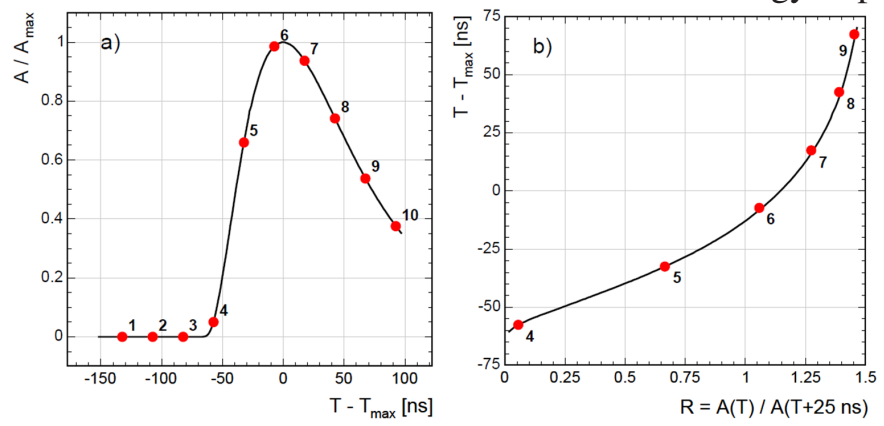

Figure 1: The reconstruction of the energy in CMS ECAL uses 10 samples of the pulse which are digitized at $40 \mathrm{MHz}$. A key design feature of the readout electronics is that the analog pulse shape if stable and reproducible to $1 \%$ among all the channels in the ECAL. The time is extracted from the ratios of these samples as explained in the

ratio of consecutive samples. Ratios containing one of the first three samples are not considered since the samples do not carry information on the pulse timing. The most sensitive ratio is the one containing the fifth sample, the one in the center of the rising edge of the pulse. This algorithm is used in the offline reconstruction of the CMS data. The results presented here are based on this time reconstruction.

The time response of each individual crystal in-situ is calibrated by equalizing the mean time response to electromagnetic clusters measured with collisions in each channel. This calibration 
is repeated every few months. It has been observed that after a power cycle of the on detector electronics the relative phase of individual readout units can be shifted by up to $1 \mathrm{~ns}$. The main goal of the recalibration is to correct for these shifts. Smaller changes in the calibration are currently being investigated in an attempt to better understand the ultimate timing capabilities of the existing ECAL. The time response is also corrected for non-linearity with a parametric correction fitted to the data. The correction is derived for the barrel and the endcap part of the ECAL separately.

\section{Timing measurement performance of the CMS ECAL}

The timing performance of the CMS ECAL has been measured in the test beam at CERN with high energy electrons. In Fig. 2 we show the result, expressed as the spread of the difference of the time measurement of two adjacent channels as a function of the effective amplitude in ADC counts. The effective amplitude of the two channels is defined as $A_{\text {eff }}=A_{1} A_{2} / \sqrt{A_{1}^{2}+A_{2}^{2}}$. The measurement has been parametrized with a functional form accounting for a statistical component of the resolution and a constant term. The constant term in this measurement was found to be 20 ps. The resolution at $20 \mathrm{GeV}$, a typical lower threshold for electrons and photons considered in the analysis of $\mathrm{Z}$ or $\mathrm{H}$ boson decays in the CMS experiment, was found to be $80 \mathrm{ps}$.

It should be noted that this measurement compares the time of two adjacent crystals exposed to the same electromagnetic shower. Possible systematic effects from shower fluctuations, the synchronization of the readout or the long term stability of the detector cancel out in this measurement. This very good performance however demonstrates that the photo detector characteristics, the shaping time and the sampling rate of $40 \mathrm{MHz}$ do not limit the performance at the level of a few $10 \mathrm{ps}$. This observation further motivated studies to explore how an upgraded calorimeter for the CMS Phase II upgrade could achieve a similar or better timing performance in-situ [5] at HL-LHC.

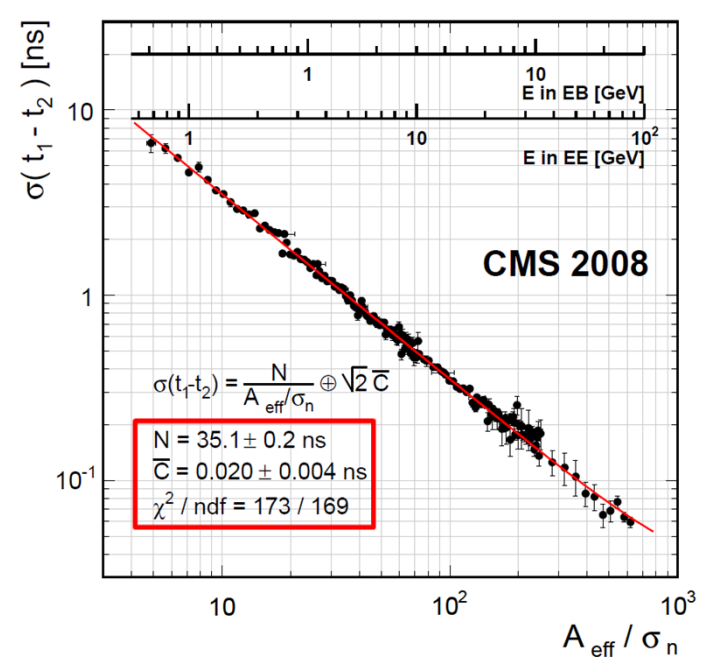

Figure 2: Timing performance of the CMS ECAL as measured in the test beam. The timing performance is expressed in the time difference measured between two channels exposed to an electromagnetic shower. The performance is shown as a function of the effective amplitude in ADC counts. The equivalent energy is shown on the top of the graph. The performance is fitted to a parametrization with a stochastic and a constant term. The constant term is estimated to be approximately $20 \mathrm{ps}$.

The timing performance of the CMS ECAL in-situ was measured with electrons from $\mathrm{Z}$ boson decays. In Figure 3 we show the relative time difference from two such electrons which we measure to be approximately $270 \mathrm{ps}$, corresponding to a single channel resolution of about $190 \mathrm{ps}$. This result was obtained with events with both electrons in the barrel. For the endcap, the corresponding single channel resolution was found to be $280 \mathrm{ps}$. The measured time was corrected for 
the path length difference of the two electrons propagating through the $3.8 \mathrm{~T}$ magnetic field of the CMS detector from the vertex to the ECAL crystals. The electron flight path is slightly curved in the magnetic field and the vertex location may vary by about $6 \mathrm{~cm}$, the RMS spread of the luminous region of the LHC accelerator inside the CMS detector. Without this path length correction the measured time difference is around 380 ps. This illustrates that even with the existing ECAL timing performance we start to be sensitive to the location of the primary vertex of the collision. The capability to reconstruct the location of the primary vertex from the time of arrival of two or more final state particles would be the target application for a precision timing detector at HL-LHC. To further investigate the difference of the measured timing precision in-situ and the performance

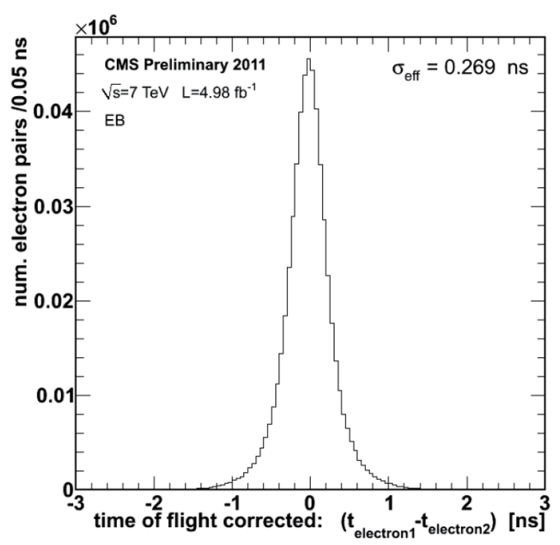

Figure 3: Time resolution as measured in $\mathrm{Z}$ boson decays into two electrons. The data used in this measurement has been accumulated during the 2010/2011 LHC data taking. The electron transverse momentum is typically $45 \mathrm{GeV}$ with a lower cut of around $20 \mathrm{GeV}$. The measured time is corrected for path length differences which can be extracted from the tracks associated with the calorimeter clusters from which the time information is extracted.

measured in the test beam we studied the timing difference of two adjacent channels in electromagnetic clusters from proton collisions in CMS. The result is shown in Figure 4. Parametrizing the energy dependence of the measured differential time resolution we find a stochastic term similar to the test beam measurements and a constant term of about $75 \mathrm{ps}$, which is significantly better than the performance measured with electrons from $\mathrm{Z}$ boson decays. A number of effects may contribute to this, such as the stability of the timing response, the calibration and the linearity of the timing response. They all may show systematic dependencies across the full detector acceptance which vanish locally, as is the case for the energy resolution.

The detector response varies under the radiation induced by the proton collisions in the detector. This is being correct for with a dedicated procedure using laser transparency measurements. The timing response is assumed to be independent of any response change of the crystals. However, the timing calibration is known to undergo long term drifts, some of which correlated with the crystal response change. This is being compensated for by repeating the in-situ time calibration about every two months. Possible correlations between the crystal response change and the timing response are being studied in more detail in the context of the work presented here. The time response correction for non-linearity is currently assumed to be invariant under the crystal response change, pending more detailed studies.

As described above, the clock distribution for the front-end electronics serves individual readout units. The clock signal must ensure that the $40 \mathrm{MHz}$ sampling remains synchronous across all channels. The exact phase of the readout units typically remains stable to much better than $1 \mathrm{~ns}$ and is reproducible for the majority of all channels after a system reset to better than $1 \mathrm{~ns}$. System resets for the front-end electronics occur only every few months, so these effects get fully compensated for by the repeated time calibrations. Smaller drifts of the phase of different readout 


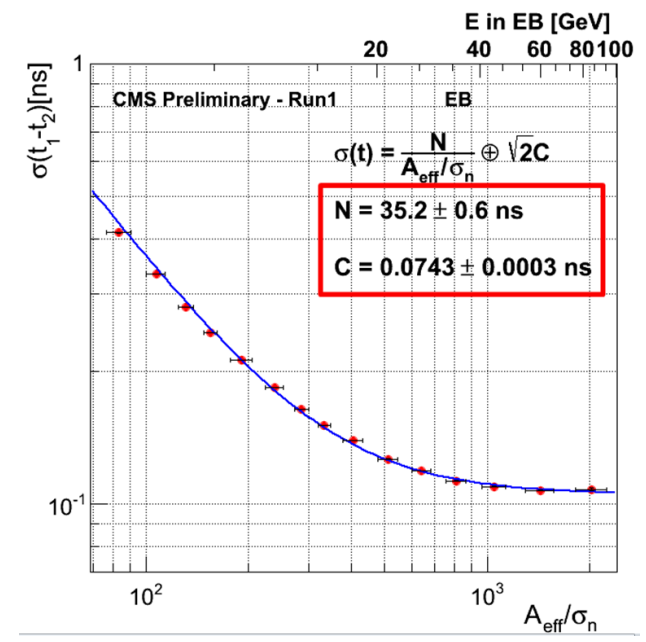

Figure 4: Time resolution as measured from two adjacent channels in a cluster from electromagnetic deposits in the ECAL. The data is fitted to a functional form accounting for stochastic and constant terms. The stochastic term matches the one measured in the testbeam, while the constant term is worse. The measured timing performance is dominated by the constant term for pulse amplitudes of about $1000 \mathrm{ADC}$ counts, corresponding to about $40 \mathrm{GeV}$. The measured resolution is significantly better than the one measured with electrons from $\mathrm{Z}$ boson decays which predominantly has equivalent energies corresponding to the flat part of the parametrization.

units may still affect the timing precision across all channels of the ECAL. In Figure 5 we show the differential timing distribution on adjacent crystals in an electromagnetic shower, restricted to crystals which share the same readout units. A further improvement of the relative time resolution to $67 \mathrm{ps}$ is observed. This provides further support to tighten the design requirements for the clock synchronization for the ECAL readout Phase II upgrades.

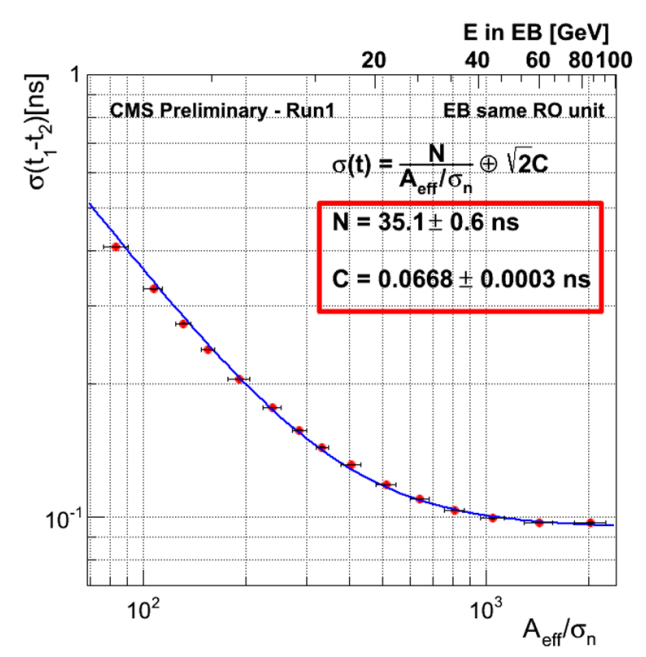

Figure 5: Time resolution as measured from two adjacent channels in a cluster from electromagnetic deposits in the ECAL. In this measurement the two channels were required to be within the same readout box, as described in the text. A further improvement of the resolution supports the evidence that the clock distribution is one of the factors contributing to the timing performance achieved in-situ.

\section{Plans and prospects for high luminosity LHC}

A high luminosity upgrade is planed for the LHC which would start data taking in approximately 2023. The target is to accumulate $3 \mathrm{ab}^{-1}$ over a time span of 10 years. LHC will operate at instantaneous luminosities in the range of $10^{35} \mathrm{~cm}^{-2} \mathrm{~s}^{-1}$. The number of simultaneous protonproton collisions (pile-up) is expected to be in the range of 140 to 200 per bunch crossing. The resulting radiation levels will pose a significant challenge on the detector technology to be deployed, in particular in the forward detector regions. The expected particle rates per unit rapidity will require upgrades to the exiting detector to maintain the current ability of identifying and precisely reconstructing physics objects such as leptons, photons and jets. 
One strategy is to increase the granularity of the detectors, in particular the forward calorimeters, to better separate individual energy deposits. The CMS collaboration is investigating two possible scenarios for a forward calorimeter upgrade. One is based on a LYSO crystal Shashlik calorimeter with tungsten as absorber. A second one uses layers of silicon as active material and tungsten as an absorber. A rebuild of the forward calorimeter allows to optimize the detector beyond what was achieved for the existing detector to better cope with pile-up conditions. Currently

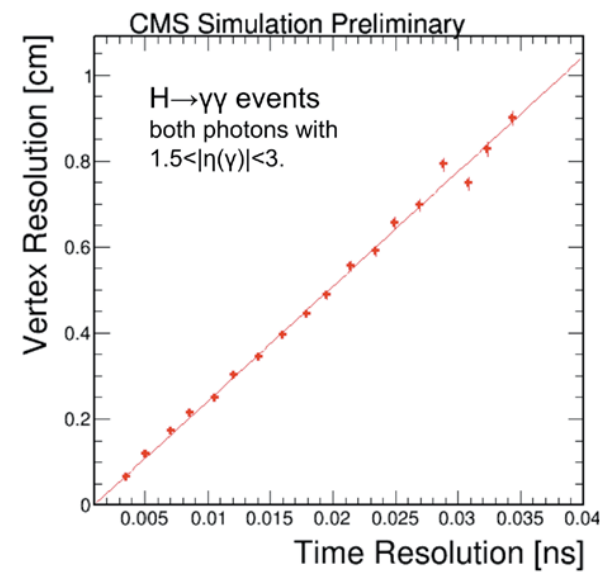

Figure 6: Simulation of the vertex location reconstruction precision for a Higgs to two photon decay using the time of arrival of the two photons in the ECAL. Knowing the time and location of arrival of the two photons, assuming that they have been produced simultaneously in the Higgs decay and knowing the $\mathrm{x}$ and $\mathrm{y}$-coordinate of the vertex, one can reconstruct the location of the third spatial coordinate. The precision with which the vertex can be reconstructed is shown as a function of the timing resolution for the two photons. With a time resolution of a few 10 ps one achieves a performance similar or better than the one achieved in the analysis of Higgs boson decay to photons with the LHC Run I data.

an extensive R\&D program is being carried out for the CMS Phase II upgrades, exploring the feasibility of the proposed forward calorimeter upgrades [7]. The potential of a precision timing detector is studied with two benchmark processes, the vertexing of photons and the jet reconstruction performance in the forward regions of the detector in a pseudo-rapidity range of 1.5 - 4.0. Various detector options for a precision timing device are being explored such as a separate detector layer capable of measuring the time of arrival of passing particles or a solution integrating a high precision timing capability into a calorimeter. Guided by the good performance of the current ECAL detector a major effort is geared towards ensuring that the ECAL is capable of achieving a timing resolution of a few $10 \mathrm{ps}$ in Phase II.

For the barrel part of the current ECAL calorimeter there are opportunities to optimize the readout to maintain or improve the current performance of the detector [8]. As demonstrated above, a significant contribution to the current performance in situ is traced to the long term stability of the clock synchronization and distribution. An increased analog bandwidth and sampling rate of the readout electronics is also being studied.

For the forward calorimeter detailed studies are ongoing to explore if the Shashlik option would allow the extraction of a precise timing signal from the LYSO crystals used as an active media in this design $[9,10]$.

From the test beam performance of the CMS ECAL we see that the stochastic term of the resolution curve is limiting the performance at low energies. Hence it would be desirable to increase the photon statistics which could be possible with LYSO, having a light yield about 300 times larger than the one for the current $\mathrm{PbWO}_{4}$ crystals. Even with a sampling fraction of about $10 \%$ and further losses due to the fibers guiding the scintillation light to the photon detectors one may increase the number of photo electrons. 


\section{Summary}

The CMS ECAL calorimeter features a very good time resolution for high energy electromagnetic deposits. A timing resolution of 190 ps has been measured for the CMS ECAL barrel with electrons from $\mathrm{Z}$ boson decays. The constant term of the timing resolution measured in the test beam for adjacent channels in a electromagnetic cluster was found to be $20 \mathrm{ps}$. Measurements of the relative timing performance in an equivalent topology from collision data yielded a constant term of the resolution of $75 \mathrm{ps}$. Studies are ongoing to further understand this difference between the performance within a single cluster and the performance across the entire detector. The long term stability of the calibration and the impact of the crystal response changes in the radiation environment at $\mathrm{LHC}$ are under investigation. Restricting the analysis to calorimeter channels sharing a common clock distribution line improves this value to $67 \mathrm{ps}$. This suggests that the clock distribution is one of the limiting factors in the timing performance achieved in-situ.

A timing resolution of a few $10 \mathrm{ps}$ would be very beneficial for the event reconstruction in the pile-up conditions expected for HL-LHC. An extensive R\&D program is currently carried out to explore the feasibility of achieving such a precision with an upgraded CMS detector. Several studies indicate that the desired performance could be achieved with a calorimeter featuring precision timing capabilities.

\section{References}

[1] S. Chatrchyan et al., CMS Collaboration, "The CMS experiment at the CERN LHC", JINST 3, S08004 (2008).

[2] L. Evans and P. Bryant, "LHC Machine", JINST 3, S08001 (2008).

[3] S. Chatrchyan et al. , CMS Collaboration, "Time Reconstruction and Performance of the CMS Electromagnetic Calorimeter", JINST 5, T03011 (2010).

[4] "Energy calibration and resolution of the CMS electromagnetic calorimeter in pp collisions at $\sqrt{7}$ TeV", CMS Collaboration, http://arxiv.org/abs/1306.2016

[5] A. Bornheim,"Evolution of the CMS ECAL response, R\&D studies on new scintillators and possible design options for electromagnetic calorimetry at the HL-LHC", Proceedings of the CHEF 2013, Paris, France, Eds. J.-C. Brient, R. Salerno, and Y. Sirois

[6] A. Martelli, "The CMS Electromagnetic Calorimeter: lessons learned during LHC run 1, overview and future projections", TIPP 2014, Amsterdam, The Netherlands, these proceedings

[7] B. Bilki, "CMS Forward Calorimetry R\&D for Phase II Upgrade", TIPP 2014, Amsterdam, The Netherlands, these proceedings

[8] M. Planer, "The CMS electromagnetic calorimeter barrel upgrade for High-Luminosity LHC", TIPP 2014, Amsterdam, The Netherlands, these proceedings

[9] A. Bornheim, "Calorimeters for precision timing measurements in high energy physics", CALOR 2014, Giessen, Germany, to be published in IOP Conference Series

[10] A. Apresyan, "Calorimeters for precision timing measurements in high energy physics", TIPP 2014, Amsterdam, The Netherlands, these proceedings 